\title{
Comptable ou visionnaire?
}

\section{Remo Osterwalder}

Dr méd., vice-président de la FMH, responsable du département Prestations et développement professionnel

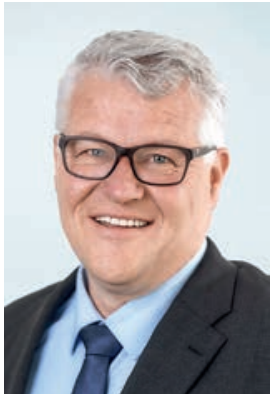

Avant tout, précisons que je porte beaucoup de respect à la profession de comptable. Sans l'aide de ces derniers, je serais tout simplement perdu pour le traitement et l'analyse des chiffres. Mais les comptables, respectivement leurs activités, illustrent de manière symbolique la situation des médecins dans le système de santé actuel. Les chiffres qu'ils gèrent correspondent à une situation en cours. En revanche, leurs analyses portent toujours sur le passé en raison du temps requis pour interpréter les chiffres. Il est certainement juste et important de procéder à de telles analyses, comme le rappelle le rapport sur notre travail quotidien établi par notre confrère Daniel Oertle [1]. Nous pouvons aussi constater que les activités sans contact direct avec les patients continuent de nous faire perdre des collègues. Mais il est tout aussi certain que nous devrions savoir pourquoi cela arrive et quelles en sont dans chaque cas les raisons.

Ces analyses ne contribuent pas à résoudre à elles seules le problème. A différents niveaux de notre système de santé, nous avons de très nombreux administrateurs qui veulent se profiler et se mettre en avant. Lorsque Stefan Felder, économiste de la santé, déclare

\section{Non seulement le visionnaire se projette dans l'avenir, mais en plus il le façonne.}

qu'il souhaite un rationnement des prestations médicales, il fait l'aveu d'une faillite: la solution ne peut en aucun cas consister à simplement refuser l'accès aux soins de santé. Cela déboucherait directement sur une médecine à deux vitesses et ne permettrait qu'à une couche restreinte de population de bénéficier d'un accès illimité à une prise en charge médicale de qualité. D'autant plus qu'il est déjà clair qui en ferait les frais. Où sont alors les autres, les visionnaires dans notre système de santé? Ils existent, nous les croisons pour ainsi dire partout: ce sont nos confrères et consœurs, d'autres professionnels de la santé, mais aussi des politiciens et des assureurs. Tous essaient avec des projets ciblés, essentiellement déployés au niveau local, de contribuer à la prise en charge médicale. Ce qu'il faut c'est une utilisation raisonnable des moyens à disposition. C'est là qu'intervient le visionnaire qui développe des modèles adaptés aux exigences de demain. La profession de médecin va changer: il est probable que l'informatique dans notre travail nous incitera à encore davantage d'algorithmes.

Les études ne sauraient constituer à elles seules une base pour les traitements car elles ne suffisent pas à couvrir réellement tous les secteurs de la médecine. Comme nous le savons, chaque étude ne permet pas de

\section{Les propositions des visionnaires ne sont pas} que des solutions unilatérales, elles s'adressent de la même manière à tous les acteurs.

répondre aux exigences requises pour se rapprocher le plus près possible de la situation idéale. Il n'y a pas que nous, médecins, qui utiliserons ces aides, mais il y aura aussi de plus en plus d'autres groupes professionnels qui prennent en charge des patients dans le secteur ambulatoire. D'une part, un gain d'efficacité doit en ressortir et d'autre part, les ressources en place pourront aussi être utilisées de manière plus ciblée. Pensons ici notamment aux listes Choosing Wisely de la Société Suisse de Médecine Interne Générale, qui pourraient alimenter des algorithmes et par conséquent être mises davantage en œuvre. Pour que de telles évolutions soient possibles, des incitatifs convaincants sont nécessaires, que ce soit pour le corps médical ou pour les patients. Ces incitatifs doivent être pensés de sorte à être intéressants pour tous les acteurs. Cela ne peut se réaliser que conjointement car des mises en application unilatérales de propositions mènent rarement à un consensus.

\footnotetext{
Références

1 Oertle E. Die Hausärzte sterben aus, na und? Bull Méd Suisses. 2016;97(51-52):1804-5.
} 